Short communication

\title{
Progranulin plasma levels as potential biomarker for the identification of GRN deletion carriers. A case with atypical onset as clinical amnestic Mild Cognitive Impairment converted to Alzheimer's disease
}

\author{
Miryam Carecchio a,b,*, Chiara Fenoglio ${ }^{a}$, Milena De Riz ${ }^{a}$, Ilaria Guidi ${ }^{c}$, Cristoforo Comi b,d, \\ Francesca Cortini ${ }^{a}$, Eliana Venturelli ${ }^{a}$, Ilaria Restelli ${ }^{a}$, Claudia Cantoni ${ }^{a}$, Nereo Bresolin ${ }^{a}$, \\ Francesco Monaco ${ }^{\text {b }}$, Elio Scarpini ${ }^{\text {a }}$, Daniela Galimberti ${ }^{a}$ \\ a Department of Neurological Sciences, “Dino Ferrari” Centre, IRCCS Fondazione Ospedale Maggiore Policlinico, University of Milan, Milan, Italy \\ b Department of Neurology, Amedeo Avogadro University, Novara, Italy \\ ' Department of Rehabilitation and Neurosciences, Neurologic Rehabilitation Division, Azienda Ospedaliera "G. Salvini", Garbagnate Milanese, Milan, Italy \\ d Neurorehabilitation Institute "M.L. Novarese", Moncrivello, VC, Italy
}

\section{A R T I C L E I N F O}

\section{Article history:}

Received 17 May 2009

Received in revised form 2 July 2009

Accepted 7 July 2009

Available online 14 August 2009

\section{Keywords:}

Progranulin

Alzheimer's disease

Mild Cognitive Impairment

Frontotemporal Lobar Degeneration

Mutation

Clinical phenotype

\begin{abstract}
A B S T R A C T
Progranulin (GRN) mutations are associated with different clinical phenotypes, including Frontotemporal Lobar Degeneration (FTLD), Corticobasal Degeneration and Alzheimer's disease (AD). In addition, the range of age at onset is very wide and patients presenting initial symptoms around eighty years have been described. Previous studies demonstrated that progranulin plasma levels determination may be a reliable method to identify GRN deletion carriers. We thus evaluated progranulin plasma levels in all patients followed at our Alzheimer's Centre whose plasma was available $(n=176)$ and found four patients displaying low values. Three of them carried the CACT deletion in exon 7 and their clinical diagnosis was behavioral variant Frontotemporal Dementia. We also identified a patient carrying a previously reported CAGT deletion in exon 5.

Here, we report on this case. The onset of symptoms was at 77 years and the initial diagnosis was of amnestic Mild Cognitive Impairment (aMCI), which converted to AD six months later. In the following years, the patient also developed behavioral disturbances, gait apraxia and parkinsonian symptoms. At present, she is 84 years old and is still followed-up periodically.

This case confirms progranulin plasma levels as a reliable biomarker to identify GRN deletion carriers and discriminate between FTLD and other dementias which may mimic it. We thus encourage the inclusion of this non-invasive and easy test in clinical practice.
\end{abstract}

(c) 2009 Elsevier B.V. All rights reserved.

\section{Introduction}

In 2006 mutations in progranulin gene (GRN) were identified to be the cause of familial Frontotemporal Lobar Degeneration with ubiquitin-positive, TAR DNA-binding protein (TARDBP, also known as TDP-43)-positive inclusions (FTLD-U). The first discovered mutations were shown to cause haploinsufficiency through nonsensemediated mRNA decay $[1,2]$. Since then, several novel mutations and variants in this gene have been found in either familial or sporadic cases (www.molgen.ua.ac.be). Notably, as opposed to previously known mutations in tau gene (MAPT), GRN mutations are associated

\footnotetext{
* Corresponding author. Department of Neurological Sciences, “Dino Ferrari” Centre, IRCCS Fondazione Ospedale Maggiore Policlinico, University of Milan, Via F. Sforza 35, 20122 Milan, Italy. Tel.: +392 55033847; fax: +39250320430.

E-mail address: mcarecchio@gmail.com (M. Carecchio).
}

with widely variable clinical phenotypes, including behavioral variant Frontotemporal Dementia (bvFTD), Progressive Aphasia (PA), Corticobasal Degeneration (CBD), Alzheimer's disease (AD), amnestic Mild Cognitive Impairment (aMCI) and Parkinson's disease (PD) (see [3] for review). Moreover, the age at onset is largely heterogeneous, ranging from 35 to 89 years, with a mean age of about 60 years [3].

Recently, it has been repeatedly demonstrated that carriers of deletions in GRN display very low plasma progranulin levels as compared with non-carriers [4-6]. As this assessment is non-invasive and easy to perform, it may be a reliable method to identify progranulin deletion carriers without sequencing the whole GRN gene, which is indeed technically more complicated and even more expensive.

Given these premises, we determined progranulin plasma levels using an ELISA kit (AdipoGene, Korea) in all patients followed at our Alzheimer's Centre from 2002 to 2008 whose plasma samples were available. Samples collected were stored at $-30{ }^{\circ} \mathrm{C}$ until use. 
A total of 176 subjects were screened, including 75 patients clinically diagnosed with $\mathrm{AD}, 45$ with bvFTD and 56 with $\mathrm{MCI}$ or subjective memory complaints. Informed consent was obtained from either patients or their caregiver.

\section{Results and clinical case description}

Four patients showed progranulin plasma levels lower than the normality reference values previously proposed [4] with a mean value of $36.24 \pm 12.55 \mathrm{ng} / \mathrm{ml}$ (Fig. 1). The mean progranulin plasma levels of the remainder screened subjects were $221.25 \pm 10.05 \mathrm{ng} / \mathrm{ml}$ in the AD group, $216,00 \pm 12.26 \mathrm{ng} / \mathrm{ml}$ in the FTLD group and $228.51 \pm 10.63 \mathrm{ng} /$ $\mathrm{ml}$ in the MCl-subjective memory complaints group (Fig. 1). Thus, we performed direct sequencing of GRN in subjects displaying low values, which demonstrated the presence of a known 4 bp deletion in GRN exon 7 (c.813_816delCACT) in three of them. All of them had a clinical diagnosis of bvFTD (range: $48-56$ years) and none showed a positive family history for dementia and/or behavioral problems. Unexpectedly, we also found a previously published [1] deletion in exon 5 (c.388_391delCAGT) in a patient clinically diagnosed with AD, whose onset was at 77 years of age. In addition, GRN gene was sequenced in all FTLD patients, as well as in subjects with plasma $G R N$ values $<150 \mathrm{ng} /$ $\mathrm{ml}$. As expected, no mutations were found in these subjects. Therefore, using the cut-off previously proposed by Ghidoni et al. [4], in accordance with their results, we had a sensitivity (defined as: real carriers/total carriers identified through the test) and specificity (real non-carriers/ total non-carriers identified through the test) of $100 \%$, supporting the usefulness of the test.

Here, we report on the case clinically diagnosed as AD. The patient came to our attention in 2003, when she was 78. She had no significant family history of neurological disorders; at that time, the patient had one brother and four sisters aged 79, 69, 74, 76, 80 years respectively and none of them had ever complained of any cognitive, behavioral or motor problem. The patient's sister aged 74 suffered from depression. Despite the apparent lack of a significant family history, the patient's mother (who died in her eighties) was reported to suffer from behavioral disturbances (although not documented), cardiovascular problems and movement difficulties due to overweight. All the patient's siblings are healthy at the moment.

Patient past medical history only revealed blood hypertension treated with an ACE-inhibitor (Ramipril $5 \mathrm{mg} /$ day). She had 12 years of education. Her daughter first noticed memory deficits and anxiety about one year prior to admission to our Department; at that time, the patient denied to have memory problems and became slightly aggressive if reminded of them. The neurological examination did not show evident deficits in cognitive functions, and was otherwise unremarkable. The patient underwent a full neuropsychological assessment, including evaluation of attention, calculation, logical reasoning, language, memory, praxis and visuospatial functions. The Mini Mental State Examination (MMSE) score

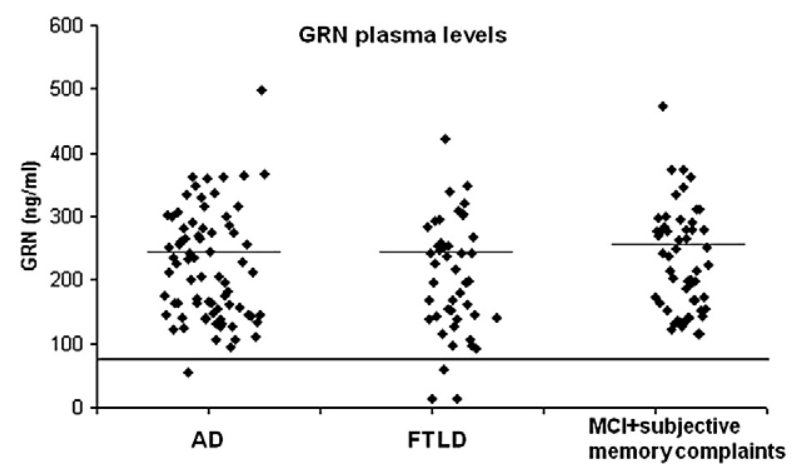

Fig. 1. Plasma progranulin levels in patients clinically diagnosed with $\mathrm{AD}(n=75)$ or bvFTD $(n=45)$ and in subjects with MCI or subjective memory complaints $(n=56)$. The black line represents the threshold value previously proposed [4]. was $27 / 30$, there was a slightly pathological performance at the Blessed A scale and borderline scores at memory tests. Blood routine analysis was normal, as well as homocysteine, folate, thyroid function and Vitamine B12 blood levels. A CT scan of the brain was performed, only showing a mild periventricular hypodensity of the white matter, which was interpreted as vascular-related in accordance with the patient's age and blood hypertension. A diagnosis of amnestic Mild Cognitive Impairment (aMCI) was made, in accordance with the criteria proposed by Petersen et al. [7] At that time, patient's plasma, serum and DNA were collected and stored at $-30{ }^{\circ} \mathrm{C}$.

She underwent a second neuropsychological assessment six months later, showing a substantial worsening in memory tasks with a MMSE of $21 / 30$. Thus, a diagnosis of probable AD was made according to NINCDSADRDA criteria [8] and she was started on Rivastigmin $1.5 \mathrm{mg}$ twice a day and Citalopram $10 \mathrm{mg}$ a day because of the presence of anxiety. Her memory complaints progressively worsened until September 2004, when she also developed apathy, problems in carrying out activities of daily living and persecutory ideations. However, MMSE was 23/30 at that time and no objective memory worsening could be detected. She was well oriented in space but not in time. The CT scan of the brain showed diffuse cortical and subcortical atrophy without focal lesions. Rivastigmin was increased up to $4.5 \mathrm{mg}$ twice a day, which caused marked daily sleepiness and was therefore suspended one month later, as no further memory complaints had occurred. In October 2005 she underwent a third neuropsychological assessment; MMSE score was 23/ 30 , with the same pattern observed about one year before (slight memory impairment and pathological score at Activities of Daily Living items). Behavioral disturbances were still present but apparently they were less severe; furthermore, the patient gained weight owing to hyperphagia and could not carry out the activities of daily living by herself, so she started being helped by a caregiver. In October 2006 her MMSE was still 22/30; persecutory ideations improved but she was markedly disoriented in time, anosognosic, progressively lost the insight into her deficits, and was almost totally unable to take care of herself. She was poorly compliant during the neurological examination and aggressive with relatives; hyperfagia was still present.

In January 2007, three years and a half after the first neurological assessment, she also developed gait apraxia and scored 18/30 at MMSE. Donepezil was started at $5 \mathrm{mg}$ once a day, and it was well tolerated.

Two years later, at age 83, the patient also started developing parkinsonian features, including reduced eye blinking, camptocormia, festinating gait, difficult swallowing, diffuse bradykinesia and rigidity in the upper limbs with cogwheel phenomenon in the left arm without resting tremor. Parkinsonian features, in particular bradykinesia and swallowing impairment, showed a significant improvement after she was started on Levodopa $300 \mathrm{mg} /$ day. At present, the patient is 84 and shows a severely reduced interaction with the environment, her spontaneous speech is almost abolished and she is able to perform only simple tasks if asked; moreover, she presents frontal release signs (glabellar and sucking reflexes).

\section{Discussion}

Results described here further confirm the usefulness of plasma progranulin level evaluation to easily detect carriers of GRN deletions, with a sensitivity and specificity of $100 \%$, in accordance with previous findings [4-6]. Moreover, we found a patient carrying a deletion in GRN exon 5 whose clinical onset was characterized by memory deficits and was initially diagnosed with aMCI and few months later with probable $\mathrm{AD}$. Her initial symptoms developed at the age of 77 and were characterized by slight memory complaints and anxiety; later, she also developed hyperphagia, persecutory ideations and extrapyramidal symptoms.

Despite the apparent lack of a significant family history in this case, the patient's mother (who died in her eighties) was reported to have behavioral disturbances and hyperphagia. This information, along with 
the patient's old age at onset, suggests an incomplete penetrance of the mutation identified.

These data confirm the observation that progranulin plasma level determination may be a reliable method to identify progranulin deletion carriers and encourage the inclusion of this non-invasive and easy test in the clinical practice. In our series, 3 out of 75 (4\%) FTLD patients screened carried the delCACT mutation in GRN exon 7, thus confirming the previous observation that this mutation is one of the most common worldwide (along with the Arg493x) [9]. This deletion could therefore be identified through progranulin plasma level evaluation, before undergoing a complete sequencing of the whole GRN gene, which implies a more complex and expensive technology.

However, it still needs to be clarified whether this method may be useful to identify carriers of single base mutations. Recently, Finch et al. analyzed a large cohort of FTD and AD patients and unaffected relatives, confirming a reduction in plasma progranulin levels in carriers of GRN loss-of-function mutations, whereas patients carrying single base mutations had partial reduction in progranulin levels, but to a lesser extent than carriers of loss-of-function mutations [6]. In our bvFTD series, we sequenced all GRN exons but did not find any carrier of single base mutations.

The biological basis explaining so low levels of progranulin in mutation carriers is unknown. Due to the haploinsufficiency mechanism, we would expect progranulin plasma levels to be $50 \%$ reduced in mutation carriers with respect to subjects carrying two coding genes, rather than few $\mathrm{ng} / \mathrm{ml}$. A possible mechanism could be a disequilibrium between progranulin and granulins occurring in patients with haploinsufficiency.

With regard to the patient carrying the deletion in GRN exon 5 , her clinical presentation was quite atypical. In fact, she presented with episodic memory disorders as the first symptom, with very mild behavioral changes, which worsened over time. Cognitive symptoms and senile onset lead clinicians to make a diagnosis of aMCI and later of $\mathrm{AD}$ in the pre-progranulin era. Nevertheless, the patient subsequently developed all the most common symptoms previously observed in carriers of GRN mutations, including behavioral disturbances, delusions, hyperphagia and parkinsonian features.

Interestingly, in our case the disease onset was in senile age. This confirms the previous observation that the range of age at onset is widely variable in GRN mutation carriers, although FTD is traditionally considered a pre-senile dementia. In a recent work by Le Ber et al., 7 out of 32 (22\%) GRN mutation carriers were older than 65 years at onset, and only 3 of them were over-70 [10].

So far, a few patients clinically diagnosed with $\mathrm{aMCI}$ or $\mathrm{AD}$ and carrying a GRN mutation have been described $[6,10,11]$. The patient described here is the first one carrying this mutation and diagnosed first as $\mathrm{aMCI}$ and then as $\mathrm{AD}$. Thus, given all findings emerged in the last couple of years and the very high sensitivity and specificity of this method, progranulin plasma level determination followed, if needed, by GRN mutation analysis should be carried out even in patients with memory disturbances when the progression of symptoms is unexpectedly slow and/or when behavioral changes and parkinsonian signs coexist during the disease course.

Extrapyramidal features have been described in association with FTD, and they can present both at the onset of the disease and in the late stages, making the clinical phenotype of GRN mutation carriers even more complex and variable, including CBD, Lewy body dementia and PD [10-13].

Parkinsonism seems to be more frequent in GRN mutations carriers than in non-carriers $[14,15]$. This may be explained with a more severe and widespread pattern of gray matter loss which has been demonstrated in GRN mutation carriers, who also show a greater degeneration of substantia nigra and basal ganglia as compared to noncarriers [14]. Our patient also showed a significant improvement of extrapyramidal symptoms after Levodopa treatment (300 mg/day), which is not a common feature in FTDP-17. In fact, the majority of patients carrying a GRN mutation usually show no or modest improvement with Levodopa $[4,16]$, although few cases of responders have been reported [17].

With regard the CT brain scan of our patient, it showed cortical and subcortical atrophy and periventricular hypodensity which was suggested to be of vascular origin, given the blood hypertension and her old age. Nevertheless, subcortical changes involving the white matter have been described in MRI brain imaging in association with GRN mutations $[3,17]$, thus the observed alterations may have been not entirely related to a vascular etiology.

Almost seven years after the onset of symptoms, the patient described here is still alive, therefore the brain pathology is not available yet, but this piece of information will be required to demonstrate the presence of ubiquitin and TDP-43 protein positive inclusions in the brain, which are considered the hallmarks of FTLD with GRN mutations.

\section{Acknowledgements}

Authors are supported by Associazione "Amici del Centro Dino Ferrari”, Monzino Foundation, IRCCS Fondazione Ospedale Maggiore (Milan) and Ing. Cesare Cusan.

\section{References}

[1] Baker M, Mackenzie IR, Pickering-Brown SM, Gass J, Rademakers R, Lindholm C, et al. Mutations in progranulin cause tau-negative frontotemporal dementia linked to chromosome 17. Nature 2006;442(7105):916-9.

[2] Cruts M, Gijselinck I, van der Zee J, Engelborghs S, Wils H, Pirici D, et al. Null mutations in progranulin cause ubiquitin-positive frontotemporal dementia linked to chromosome 17q21. Nature 2006;442(7105):920-4.

[3] van Swieten JC, Heutink P. Mutations in progranulin (GRN) within the spectrum of clinical and pathological phenotypes of frontotemporal dementia. Lancet Neurol 2008;7 (10):965-74.

[4] Ghidoni R, Benussi L, Glionna M, Franzoni M, Binetti G. Low plasma progranulin levels predict progranulin mutations in Frontotemporal Lobar Degeneration. Neurology 2008;71(16):1235-9.

[5] Finch N, Baker M, Crook R, Swanson K, Kuntz K, Surtees R, et al. Plasma progranulin levels predict progranulin mutation status in frontotemporal dementia patients and asymptomatic family members. Brain 2009;132(Pt 3):583-91.

[6] Sleegers K, Brouwers N, Van Damme P, Engelborghs S, Gijselinck I, van der Zee J, et al. Serum biomarker for progranulin-associated Frontotemporal Lobar Degeneration. Ann Neurol 2009;65(5):603-9.

[7] Petersen RC, Smith GE, Waring SC, Ivnik RJ, Tangalos EG, Kokmen E. Mild Cognitive Impairment: clinical characterization and outcome. Arch Neurol 1999;56(3):303-8.

[8] McKhann G, Drachman DA, Folstein M, Katzman R, Price DL, Stadlan EM. Clinical diagnosis of Alzheimer's disease - report of the NINCDS-ADRDA work group under the auspices of Department of Health and Human Services Task Force on Alzheimer's disease. Neurology 1984;34:939-44.

[9] Benussi L, Ghidoni R, Pegoiani E, Moretti DV, Zanetti O, Binetti G. Progranulin Leu271LeufsX10 is one of the most common FTLD and CBS associated mutations worldwide. Neurobiol Dis 2009;33(3):379-85.

[10] Le Ber I, Camuzat A, Hannequin D, Pasquier F, Guedj E, Rovelet-Lecrux A, et al. Phenotype variability in progranulin mutation carriers: a clinical, neuropsychological, imaging and genetic study. Brain 2008;131(Pt 3):732-46.

[11] Brouwers N, Nuytemans K, van der Zee J, Gijselinck I, Engelborghs S, Theuns J, et al. Alzheimer and Parkinson diagnoses in progranulin null mutation carriers in an extended founder family. Arch Neurol 2007;64(10):1436-46.

[12] Rovelet-Lecrux A, Deramecourt V, Legallic S, Maurage CA, Le Ber I, Brice A, et al. Deletion of the progranulin gene in patients with Frontotemporal Lobar Degeneration or Parkinson disease. Neurobiol Dis 2008;31(1):41-5.

[13] Benussi L, Binetti G, Sina E, Gigola L, Bettecken T, Meitinger T, Ghidoni R. A novel deletion in progranulin gene is associated with FTDP-17 and CBS. Neurobiol Aging 2008;29(3):427-35.

[14] Josephs KA, Ahmed Z, Katsuse O, Parisi JF, Boeve BF, Knopman DS, et al. Neuropathologic features of Frontotemporal Lobar Degeneration with ubiquitinpositive inclusions with progranulin gene (PGRN) mutations. J Neuropathol Exp Neurol 2007;66(2):142-51.

[15] Whitwell JL, Jack Jr CR, Baker M, Rademakers R, Adamson J, Boeve BF, et al. Voxelbased morphometry in Frontotemporal Lobar Degeneration with ubiquitin-positive inclusions with and without progranulin mutations. Arch Neurol 2007;64(3):371-6.

[16] Boeve BF, Baker M, Dickson DW, Parisi JE, Giannini C, Josephs KA, et al. Frontotemporal dementia and parkinsonism associated with the IVS1 $+1 \mathrm{G}>\mathrm{A}$ mutation in progranulin: a clinicopathologic study. Brain 2006;129:3103-14.

[17] Kelley BJ, Haidar W, Boeve BF, Baker M, Graff-Radford NR, Krefft T, et al. Prominent phenotypic variability associated with mutations in Progranulin. Neurobiol Aging 2009;30(5):739-51. 